\title{
5 years later: irisin detection still an issue
}

\author{
Alexandra B Cooke', Yessica-Haydee Gomez ${ }^{2}$ and Stella S Daskalopoulou, ${ }^{1,2}$ \\ ${ }^{1}$ Division of Experimental Medicine, Department of Medicine, Faculty of Medicine, McGill University, Montreal, \\ Quebec, Canada and ${ }^{2}$ Division of Internal Medicine, Faculty of Medicine, Research Institute of the McGill University \\ Health Centre, Montreal, Quebec, Canada
}

\author{
Correspondence \\ should be addressed \\ to S S Daskalopoulou \\ Email \\ stella.daskalopoulou@mcgill.ca
}

Since the discovery of irisin in early 2012 (1), considerable attention has been directed toward understanding the physiological role of this circulating myokine in humans. Animal studies demonstrating important metabolic benefits have been convincing $(1,2)$, but whether these findings translate to humans remains unclear, and many discrepant results have been reported $(3,4,5)$. A tremendous limiting factor continues to be the quality and accuracy of existing commercial enzymelinked immunosorbent assays (ELISAs) for quantifying circulating irisin levels. With growing interest in this field, resulting in over 500 publications since 2012, we feel it is important to reinforce some of the limitations of the currently available assays and the necessary steps forward, as well as share our own concerning results regarding the Phoenix Pharmaceuticals and Adipogen ELISAs, two of the most widely used assays to date (3).

Our group intended to quantify circulating irisin levels in young healthy adults in response to exercise; however, our own validation testing with both ELISAs has been less than optimal. Using the $1^{\text {st }}$-generation Phoenix Pharmaceuticals EK-067-29 assay, we observed very high coefficients of variation (CVs) of $13.2 \%$ and $13.8 \%$ for 2 separate human plasma samples (17 duplicates each, loaded interchangeably across the plate). Phoenix Pharmaceuticals subsequently improved the specificity of the biotinylated peptide in the 2nd generation of this assay (current version), yielding concentrations much closer to the 'true' circulating irisin levels, determined using tandem mass spectrometry (6). While this seemed a positive development, we remain dissatisfied with the performance of this assay, reporting CVs of $15.2 \%$ and $11.0 \%$ for 2 samples (13 duplicates each). We further tested the same 2 samples with the Adipogen ELISA (AG-45A0046YEK-KI01) (10 duplicates each) and observed even higher CVs of $21.4 \%$ and $11.1 \%$ (Table 1). It should be emphasized that the irisin concentrations obtained from the Adipogen assay are considerably higher than the expected range (6): irisin values measured with the Adipogen assay were 477 -fold higher than those of the Phoenix assay in the same individual (Table 1).

Irisin secretion is thought to be regulated by exercise; however, many studies have reported contradictory results (3). This is not surprising given the high levels of inter- and intra-assay variation with these assays. In our lab, we observed large differences in the percent increase in irisin levels after acute maximal exercise in 4 healthy individuals between the original EK-067-52 assays (30.8 $\pm 27.6 \%$ increase) (7) and 2 different EK-067-29 assays within the same lot $(2.6 \pm 10.2 \%$ and $16.2 \pm 34.5 \%)$ (single freeze-thaw cycle). Montes-Nieto et al. have also reported issues with the EK-067-29 assays, showing inconsistent results between the $1^{\text {st }}$ - and $2^{\text {nd }}$-generation versions: a relationship between excess weight and irisin levels was noted with the $1^{\text {st }}$, but not the $2^{\text {nd }}$-generation assay (8). We also observed notable differences in the relative increase detected with the Phoenix Pharmaceutical and Adipogen ELISAs (7 vs 18\%, respectively) in the same sample (Table 1). Other groups have relayed similar concerns regarding the performance of the existing ELISAs $(3,5,9)$; yet, most groups continue using these assays without appropriate validation.

Results from a recombinant spike and recovery test conducted in-house by Phoenix Pharmaceuticals using the EK-067-29 assay (personal communication) provide further corroborating evidence for these discrepant findings and other inconsistencies in the literature to date. Percent recovery at 2 concentrations of recombinant irisin was $70 \%(1 \mathrm{ng} / \mathrm{mL})$ and $115 \%(2 \mathrm{ng} / \mathrm{mL})$, falling outside the commonly accepted range of $90-110 \%$ (10). The source of this variability remains unclear. Unfortunately, validation of the specificity of the antibody by mass spectrometry analysis using human plasma/serum samples is currently lacking, as only spiked recombinant irisin has been www.eje-online.org DOI: 10.1530/EJE-17-0572
() 2017 European Society of Endocrinology Printed in Great Britain
Published by Bioscientifica Ltd. 
Table 1 Intra-assay validation testing using Pheonix Pharmaceuticals (2nd generation) and Adipogen ELISAs.

\begin{tabular}{|c|c|c|c|c|}
\hline \multirow[b]{2}{*}{ Duplicate } & \multicolumn{2}{|c|}{ Phoenix pharmaceuticals EK-067-29 (2nd generation) } & \multicolumn{2}{|c|}{ Adipogen AG-45A-0046YEK-KI01 } \\
\hline & $\begin{array}{l}\text { Sample A (pre-exercise) irisin } \\
\text { concentration }(\mathrm{ng} / \mathrm{mL})\end{array}$ & $\begin{array}{l}\text { Sample B (post-exercise) irisin } \\
\text { concentration }(\mathrm{ng} / \mathrm{mL})\end{array}$ & $\begin{array}{c}\text { Sample A (pre-exercise) } \\
\text { irisin concentration ( } \mathrm{ng} / \mathrm{mL} \text { ) }\end{array}$ & $\begin{array}{c}\text { Sample B (post-exercise) } \\
\text { irisin concentration ( } \mathrm{ng} / \mathrm{mL} \text { ) }\end{array}$ \\
\hline 1 & 11.17 & 12.50 & 4724.10 & 6846.10 \\
\hline 2 & 12.99 & 12.56 & 4578.00 & 6276.50 \\
\hline 3 & 10.80 & 11.25 & 5091.80 & 6719.30 \\
\hline 4 & 13.33 & 13.17 & 5874.80 & 6108.20 \\
\hline 5 & 13.44 & 13.67 & 4964.40 & 6164.00 \\
\hline 6 & 12.83 & 14.80 & 8455.60 & 6403.60 \\
\hline 7 & 8.97 & 9.58 & 5608.70 & 5822.20 \\
\hline 8 & 12.86 & 14.17 & 4991.20 & 7264.60 \\
\hline 9 & 12.10 & 13.38 & 5516.20 & 8412.30 \\
\hline 10 & 13.56 & 13.84 & 7123.50 & 6910.90 \\
\hline 11 & 9.17 & 12.52 & - & - \\
\hline 12 & 14.25 & 13.41 & - & - \\
\hline 13 & 9.47 & 11.33 & - & - \\
\hline Range & (8.98-14.25) & $(9.58-14.80)$ & (4578.0-8455.6) & (5822.2-8412.3) \\
\hline Mean (S.D.) & $11.92(1.81)$ & $12.78(1.41)$ & $5692.90(1216.38)$ & $6692.77(744.74)$ \\
\hline CV & $15.2 \%$ & $11.0 \%$ & $21.4 \%$ & $11.1 \%$ \\
\hline $\begin{array}{l}\text { Mean relative } \\
\text { increase } \\
\text { post-exercise }\end{array}$ & $8 \pm 11 \%$ & & $22 \pm 25 \%$ & \\
\hline
\end{tabular}

assessed to date $(2,4)$. Therefore, whether this antibody is truly specific for the different fragments of endogenous irisin has not been established.

While the Phoenix Pharmaceutical and Adipogen ELISAs are most commonly used (3), many other commercial assays utilizing polyclonal antibodies are available (Table 2). Differences in the reported range of irisin levels $(0.02-4300 \mathrm{ng} / \mathrm{mL})(11,12)$ and level of validation testing limit comparison between studies. These issues compromise our ability to extract accurate information about irisin regulation in humans as well as its role in metabolic health and other disease states. Through personal communication with various commercial assay vendors and manufacturers, we obtained additional information regarding the validation testing performed for each of the assays (Table 2). Although most commercial assays have undergone a series of in-house validation tests and have released results that meet acceptable standards, we have demonstrated significant differences in our validation tests with the Phoenix Pharmaceutical and Adipogen assays. This unfortunately is not uncommon: variable standards in antibody validation by both suppliers and researchers have triggered a widespread 'reproducibility crisis' (13), leading to conflicting results and a waste of time and money. Therefore, we would urge researchers to be cautious and conduct validation testing before running samples for study analyses.
Developing a monoclonal antibody specific for irisin is an important research priority. Both Phoenix Pharmaceuticals and Adipogen assays are competitive ELISAs with polyclonal antibodies, which are known to be less sensitive and specific, compared to a sandwich ELISA (14). In late 2016, Aviscera Bioscience developed the first sandwich ELISA with a monoclonal antibody; however, they have not verified the specificity of the antibody against human irisin in plasma/serum samples with Western blot or other established methods (personal communication). Moreover, neither the recovery nor the sensitivity of the Aviscera Bioscience assay has been evaluated, and the intra- and inter-assay reproducibility has not been appropriately assessed with human plasma/ serum samples.

Overall, numerous inconsistencies in the literature underscore the need to develop more accurate methods and perform more rigorous validation testing with different assays, ensuring the results meet established standards. We urge other researchers to consider these arguments; otherwise, we risk introducing further controversy and uncertainty by using unreliable assays. Confirming whether irisin will live up to its expectations in Men's Fitness as a 'Miracle Weight Loss Pill (that) Allows for Easy Workouts' (15) will require more reliable methodologies and scientific rigor than currently exists. 


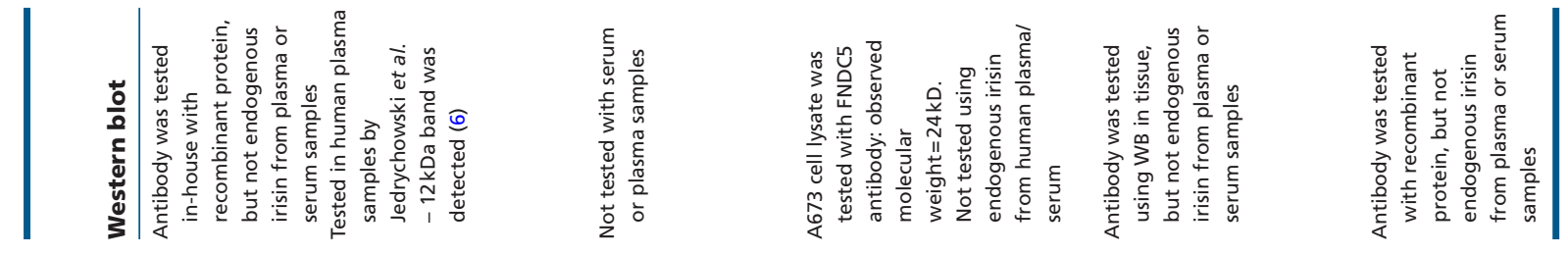

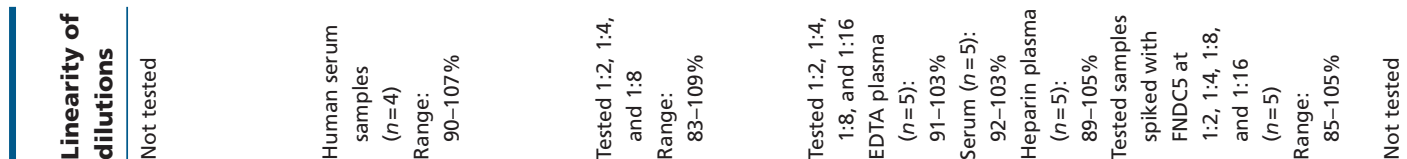

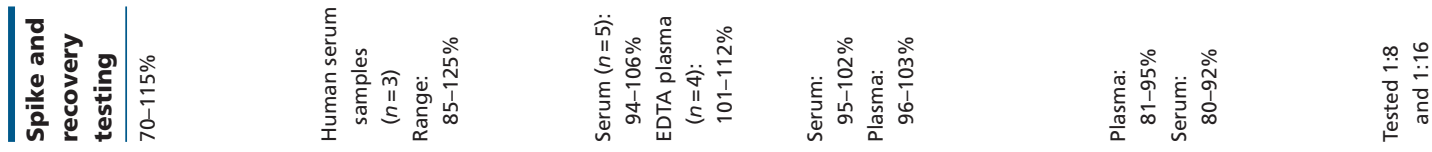

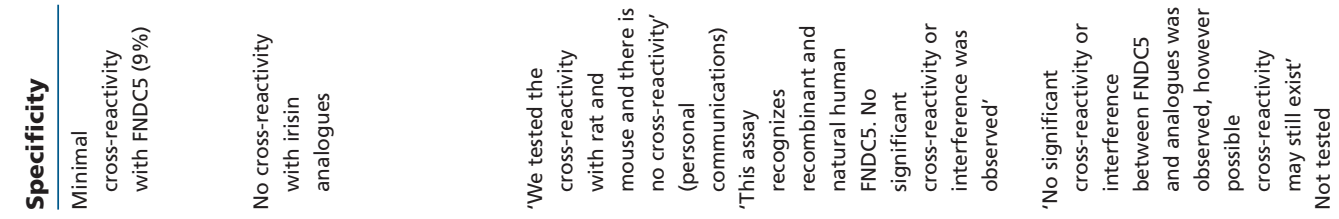

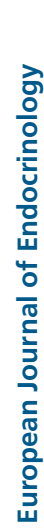

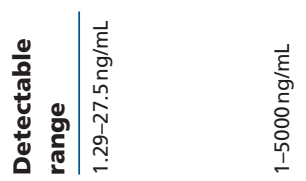

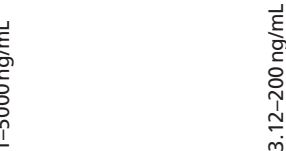

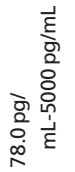

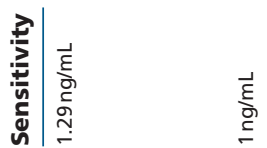

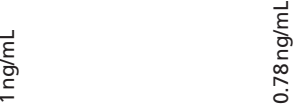

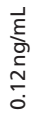

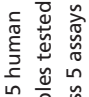

产

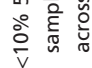

产总

$\stackrel{\substack{\circ \\ \infty}}{\substack{\infty \\ \text { in }}}$

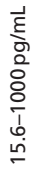

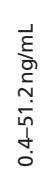

$\overrightarrow{5}$
जू
बे
bे

$\frac{\vec{\xi}}{\stackrel{\xi}{\frac{g}{0}}}$

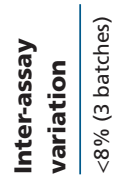

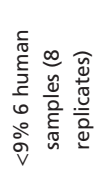

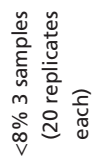

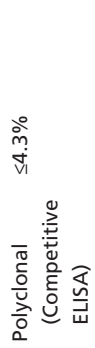

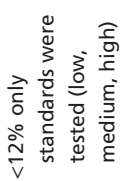

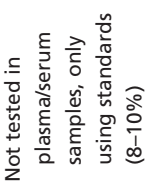

$\mid$

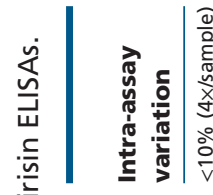

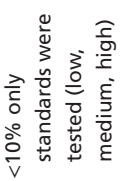

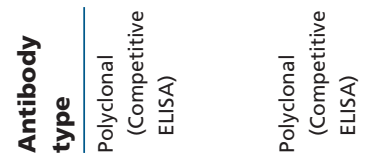

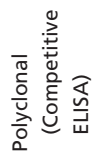

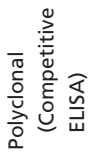

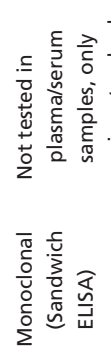

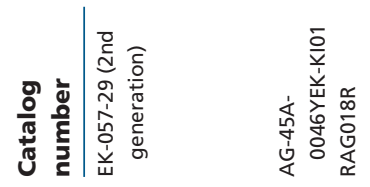

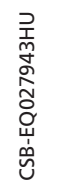

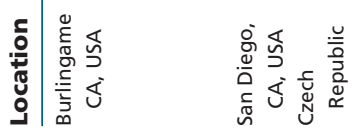

站

공

产

oे
$\dot{1}$
$\hat{े}$
ò

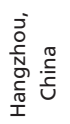

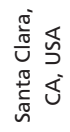

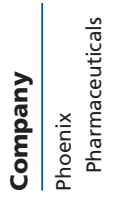

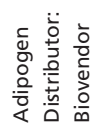

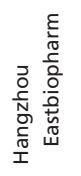

嘸

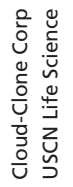

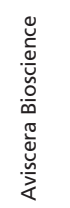

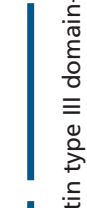

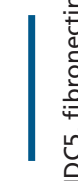

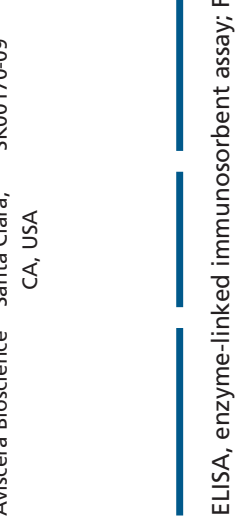




\section{Declaration of interest}

The authors declare that there is no conflict of interest that could be perceived as prejudicing the impartiality of the commentary reported.

\section{Funding}

This research did not receive any specific grant from any funding agency in the public, commercial or not-for-profit sector.

\section{Acknowledgements}

The authors would like to thank Adipogen for providing us with a complimentary trial ELISA.

\section{References}

1 Bostrom P, Wu J, Jedrychowski MP, Korde A, Ye L, Lo JC, Rasbach KA, Bostrom EA, Choi JH, Long JZ et al. A PGC1-alpha-dependent myokine that drives brown-fat-like development of white fat and thermogenesis. Nature 2012481 463-468. (doi:10.1038/nature10777)

2 Zhang Y, Li R, Meng Y, Li S, Donelan W, Zhao Y, Qi L, Zhang M, Wang $\mathrm{X}$, Cui $\mathrm{T}$ et al. Irisin stimulates browning of white adipocytes through mitogen-activated protein kinase p38 MAP kinase and ERK MAP kinase signaling. Diabetes 201463 514-525. (doi:10.2337/db13-1106)

3 Perakakis N, Triantafyllou GA, Fernandez-Real JM, Huh JY, Park KH, Seufert J \& Mantzoros CS. Physiology and role of irisin in glucose homeostasis. Nature Reviews Endocrinology 201713 324-337. (doi:10.1038/nrendo.2016.221)

4 Polyzos SA \& Mantzoros CS. An update on the validity of irisin assays and the link between irisin and hepatic metabolism. Metabolism 2015 64 937-942. (doi:10.1016/j.metabol.2015.06.005)

5 Sanchis-Gomar F, Alis R \& Lippi G. Circulating irisin detection: Does it really work? Trends in Endocrinology and Metabolism 201526 335-336. (doi:10.1016/j.tem.2015.05.004)

6 Jedrychowski MP, Wrann CD, Paulo JA, Gerber KK, Szpyt J, Robinson MM, Nair KS, Gygi SP \& Spiegelman BM. Detection and quantitation of circulating human irisin by tandem mass spectrometry. Cell Metabolism 201522 734-740. (doi:10.1016/j.cmet.2015.08.001)

7 Daskalopoulou SS, Cooke AB, Gomez YH, Mutter AF, Filippaios A, Mesfum ET \& Mantzoros CS. Plasma irisin levels progressively increase in response to increasing exercise workloads in young, healthy, active subjects. European Journal of Endocrinology 2014171 343-352. (doi:10.1530/EJE-14-0204)

8 Montes-Nieto R, Martinez-Garcia MA, Luque-Ramirez M \& EscobarMorreale HF. Differences in analytical and biological results between older and newer lots of a widely used irisin immunoassay question the validity of previous studies. Clinical Chemistry and Laboratory Medicine 201654 e199-e201. (doi:10.1515/cclm-2015-1071)

9 Sanchis-Gomar F, Alis R, Pareja-Galeano H, Romagnoli M \& Perez-Quilis C. Inconsistency in circulating irisin levels: what is really happening? Hormone and Metabolic Research 201446 591-596. (doi:10.1055/s-0033-1363283)

10 Wild D. The Immunoassay Handbook Theory and Applications of Ligand Binding, ELISA and Related Techniques. 2013. Kidlington, Oxford, UK: Elsevier Ltd.

11 Choi YK, Kim MK, Bae KH, Seo HA, Jeong JY, Lee WK, Kim JG, Lee IK \& Park KG. Serum irisin levels in new-onset type 2 diabetes. Diabetes Research and Clinical Practice 2013100 96-101. (doi:10.1016/j. diabres.2013.01.007)

12 Lawson EA, Ackerman KE, Slattery M, Marengi DA, Clarke H \& Misra M. Oxytocin secretion is related to measures of energy homeostasis in young amenorrheic athletes. Journal of Clinical Endocrinology and Metabolism 201499 E881-E885. (doi:10.1210/jc.2013-4136)

13 Baker M. Reproducibility crisis: blame it on the antibodies. Nature 2015521 274-276. (doi:10.1038/521274a)

14 Cox KL, Devanarayan V, Kriauciunas A, Manetta J, Montrose C \& Sittampalam S. Immunoassay methods. In Assay Guidance Manual. Eds GS Sittampalam, NP Coussens, K Brimacombe, A Grossman, M Arkin, D Auld, C Austin, J Baell, B Bejcek, TDY Chung, et al. Bethesda (MD): Eli Lilly \& Company and the National Center for Advancing Translational Sciences, 2004.

15 Men's Fitness. Miracle weight loss pill irisin allows for easy workouts. Accessed on 7 July 2017. Retrieved from: http://www.mensfitness. com/nutrition/supplements/miracle-weight-loss-pill-irisin-allowseasy-workouts

Received 11 July 2017

Revised version received 11 July 2017

Accepted 14 July 2017 